\title{
Das DGPPN-Zertifikat Forensische Psychiatrie
}

\author{
Entwicklung, gegenwärtige Situation, Perspektive
}

\author{
Jürgen L. Müller · N. Saimeh
}

Eingegangen: 30. Juli 2012 / Angenommen: 24. August 2012 / Online publiziert: 6. Oktober 2012

(C) Die Autor(en) 2012. Dieser Artikel ist auf Springerlink.com mit Open Access verfügbar

Zusammenfassung Die Einführung des Zertifikats Forensische Psychiatrie der Deutschen Gesellschaft für Psychiatrie, Psychotherapie und Nervenheilkunde (DGPPN) trug wesentlich zur Qualitätsverbesserung forensisch psychiatrischer Expertisen bei. Seit 2004 sind die entsprechenden Schwerpunktbezeichnungen der Landesärztekammern geschaffen und inzwischen etabliert. Gegenwärtig stehen zwei Nachweise über eine durchlaufene forensisch psychiatrische Qualifizierung nebeneinander. Dies eröffnet die Chance, das Zertifikat fortzuentwickeln und Standards für die Qualitätssicherung zu setzen. Diskutiert werden insbesondere Maßnahmen der Qualitätskontrolle, Möglichkeiten auf gravierende Qualitätsmängel zu reagieren und die Weiterbildung im Sinne eines Continuing-Medical-Education(CME)-Prozesses zu verstetigen. Bei Verankerung und Durchsetzung dieser Standards kommt der DGPPN eine Schlüsselposition zu.

Schlüsselwörter Zertifikat Forensische Psychiatrie · Qualitätssicherung $\cdot$ Schwerpunktbezeichnung

\section{The DGPPN certificate for forensic psychiatry}

Development, present situation and perspectives

Prof. Dr. J. L. Müller $(\bowtie)$

Forensische Psychiatrie und Psychotherapie der Universität Göttingen, Rosdorfer Weg 70, 37070 Göttingen, Deutschland

E-Mail: ju.mueller@asklepios.com

Dr. N. Saimeh

LWL Zentrum für Forensische Psychiatrie Lippstadt Eickelborn, Eickelborn, Deutschland
Abstract The introduction of the German Society for Psychiatry Psychotherapy and Mental Health (DGPPN) certificate for forensic psychiatry significantly contributed to improving the quality of forensic psychiatric expertises. The corresponding designations of state medical boards were created in 2004 and have now become established. Currently two methods of certification of forensic psychiatric training are possible which opens up the opportunity to further develop the certificate and to set standards for quality assurance. This article discusses in particular quality control measures, ways to respond to serious quality issues and the development process in terms of a continuing medical education. By establishing and enforcing such standards the DGPPN will achieve a key position.

Keywords Certificate forensic psychiatry · Quality assurance $\cdot$ Priority designation

\section{Situation vor Einführung des Zertifikats}

Forensisch psychiatrische Gutachten haben in den zurückliegenden Jahrzehnten ein deutlich gestiegenes Gewicht bei Fragestellungen auf allen Rechtsgebieten erlangt. Von besonderer öffentlicher Aufmerksamkeit sind dabei seit jeher Begutachtungen auf strafrechtlichem Gebiet, insbesondere zur Beurteilung der Schuldfähigkeit und der Gefährlichkeit. Letztere sind notwendig im erkennenden Strafverfahren bei der Anordnung einer Maßregel, bei der Beurteilung von Therapiefortschritten und der Lockerungseignung, bei der Beurteilung der Entlassbarkeit aus lebenslanger Haft, aus der Sicherungsverwahrung oder dem psychiatrischen Maßregelvollzug. Sachverständige werden von den Auftraggebern eigenständig bestellt. Nicht zuletzt abhängig von der durchlaufenen spezifisch forensischen Weiterbildung 
und praktischen Erfahrung, ist die Qualität dieser Expertisen sehr unterschiedlich und führte und führt immer wieder auch zu berechtigten Klagen. Durch die Einführung des Zertifikats Forensische Psychiatrie der Deutschen Gesellschaft für Psychiatrie, Psychotherapie und Nervenheilkunde (DGPPN) sowie in der Folge der Schwerpunktbezeichnung Forensische Psychiatrie der Landesärztekammern wurden wichtige Maßnahmen zur Qualitätssteigerung forensischer Gutachten etabliert. Der Einführung der Weiterbildung Forensische Psychiatrie vorangegangen war eine intensive Diskussion in den 1990er Jahren um eine notwendige Qualitätssteigerung forensisch psychiatrischer Gutachten; diese wurde durch verschiedene Impulse angestoßen:

1. Mehrere wissenschaftliche Analysen forensisch psychiatrischer Gutachten hatten z. T. erhebliche Mängel der untersuchten Expertisen offenbart [5, 12]. Qualitätsmängel und Qualitätsverbesserungen waren zu Dauerthemen des juristisch psychiatrischen Dialogs geworden. Führende Fachwissenschaftler sprachen in den 1980er Jahren gar von einem „Notstand der forensischen Psychiatrie" $[7,8,11]$.

2. Forensisch psychiatrische Kliniken klagten zunehmend über Fehleinweisungen krimineller, aber psychisch nicht erheblich gestörter Täter, die infolge ihrer verfestigten subkulturell geprägten Interaktionen das spezifisch therapeutische Milieu der Kliniken zu untergraben drohten. Gleichzeitig wurde innerhalb der interdisziplinären forensisch psychiatrischen Arbeitsgemeinschaften diskutiert, ob die steigenden Verweildauern in der forensischen Psychiatrie nicht auch Folge einer großen Unsicherheit in der Risikoprofilerstellung sind.

3. Entgegen dem gesellschaftlichen Gewicht war die universitäre Bedeutung der forensischen Psychiatrie eher gesunken. Die Steigerung des Qualitätsstandards und die Qualitätssicherung sind aber an die Kapazitäten und das Leistungsvermögen verfügbarer Ausbildungs- und Forschungsinstitute gebunden. Dementsprechend kritisierte Förster in seinen Übersichten zur Weiterbildungssituation die zu geringe Zahl und die mangelhafte Ausstattung universitärer Einrichtungen, welche für die Fort- und Weiterbildung in forensischer Psychiatrie und Psychotherapie zuständig sein können [3, 4].

4. Eine wesentliche Folge spektakulärer Sexualverbrechen an Kindern in den 1990er Jahren im In- und Ausland (Kim K. und Nathalie A. in Deutschland, der Fall Dutroux in Belgien) war die Zunahme des Sicherheitsund Sicherungsgedankens im Strafrecht und, damit verbunden, auch der Ruf nach wirksameren Therapieverfahren sowie nach einer deutlichen Professionalisierung und Qualitätssteigerung der vorgelegten Gutachten.

Der gewachsene Stellenwert, der den Expertisen und der forensischen Psychiatrie beigemessen wurde, und die gestiegenen Erwartungen gingen mit kritischen Bewertungen nach Zwischenfällen einher. Zugleich hatte sich die forensische Psychiatrie zunehmend spezialisiert und hatte, nicht zuletzt über die gewachsene Bedeutung der Behandlung psychisch kranker Rechtsbrecher, deutlich an Kontur gewonnen. Festgestellte und angeprangerte Qualitätsmängel psychiatrischer Sachverständigentätigkeit und die gewachsene Bedeutung der Behandlung psychisch kranker Rechtsbrecher machten formale und inhaltliche Qualitätsstandards für die forensisch psychiatrische Tätigkeit unverzichtbar.

\section{Entwicklung des DGPPN-Zertifikats}

Die DGPPN griff diese Diskussion aktiv auf und betonte, dass die im forensisch psychiatrischen Kontext relevanten Diagnosen und Tatkonstellationen wie sexuelle Deviationen, Persönlichkeitsstörungen, Affektdelikte und die spezifischen Aufgaben der forensischen Psychiatrie ein eigenes fachliches Profil begründen und eine differenzierte Ausund Weiterbildung zusätzlich zu der der Allgemeinpsychiatrie erforderlich machen. Dem sollte in einer spezifischen forensisch psychiatrischen Qualifikation Rechnung getragen werden. Umstritten waren neben Umfang und Ausgewogenheit der theoretischen Weiterbildung auf straf- und zivilrechtlichem Gebiet, insbesondere die erforderliche Zahl von Gutachten und deren Supervision sowie die (Un-) Entbehrlichkeit einer praktischen Tätigkeit im Maßregelvollzug. Bei einer sehr kontrovers geführten Diskussion wurden ein ebenso ausgewogenes wie hohes Niveau favorisiert und die Notwendigkeit therapeutischer Erfahrungen hervorgehoben [9, 10]. Mit dem Zertifikat Forensische Psychiatrie wurden mit konkret benannten Weiterbildungsinhalten die geforderten Qualitätsstandards der forensisch psychiatrischen Begutachtung geschaffen. Das Zertifikat Forensische Psychiatrie gewährleistete, dass die veröffentlichten konkreten Anforderungen und Ausbildungsinhalte abgeleistet wurden [11]. Mit der Zertifizierung, die nach der Einführung einer Schwerpunktbezeichnung durch die Bundes- und Landesärztekammern in eine Schwerpunktbezeichnung forensische Psychiatrie übergehen sollte, wurden die Qualitätsstandards der Gutachten deutlich angehoben, die Auftraggeber bei der Benennung fachlich qualifizierter Sachverständiger unterstützt und eine deutliche Qualitätsverbesserung von Gutachten erreicht.

\section{Inhalte der Zertifizierung}

Die Spezialisierung „Forensische Psychiatrie“ vermittelt spezifisch forensisch psychiatrische Inhalte, die in der allgemeinpsychiatrischen Weiterbildung weniger intensiv abgedeckt werden. Dazu gehören die relevanten gesetz- 
lichen Grundlagen, die Klärung der Rolle als Sachverständiger, spezifische Kenntnisse der kriminologischen Bedeutung unterschiedlicher psychiatrischer Störungsbilder, einschließlich sexueller Paraphilien, die Zuordnung von medizinischen Diagnosen zu den juristischen Eingangsmerkmalen gemäß $\S 20 \mathrm{StGB}$, die spezifische Beurteilung einzelner Deliktgruppen wie z. B. Sexualstraftaten oder Affektdelikte unter spezifisch psychiatrischen Gesichtspunkten sowie insbesondere die Vermittlung der Methoden zur Erstellung von Kriminalprognosen. Darüber hinaus werden Methoden zur gezielten Veränderung delinquenter Verhaltensmuster von psychisch gestörten Rechtsbrechern und Methoden zur Prävention künftigen delinquenten Verhaltens thematisiert. Für das Zertifikat der DGPPN wurde eine Weiterbildungszeit von 2 Jahren nach Abschluss der Weiterbildung zum Gebietsarzt für Psychiatrie und Psychotherapie bzw. Psychiatrie bzw. Nervenheilkunde in einer Einrichtung, deren Leiter zur Weiterbildung in forensischer Psychiatrie ermächtigt ist, gefordert [11]. Die Inhalte und Ziele bezogen sich auf folgende Aufgaben:

- Beurteilung der Schuldfähigkeit,

- Grundlagen der Einweisung in den Maßregelvollzug, einschließlich subsidärer Maßnahmen,

- Beurteilung der Rückfall- und Gefährlichkeitsprognose,

- Beurteilung der Verhandlungsfähigkeit,

- Beurteilung der Haftfähigkeit,

- Beurteilung der Vernehmungsfähigkeit,

- Beurteilung der Reife von Heranwachsenden nach § 105 des Jugendgerichtsgesetzes (JGG),

- zivilrechtliche Fragestellungen wie Geschäftsfähigkeit, Testierfähigkeit, Prozessfähigkeit, Begutachtungen im Rahmen des Betreuungsrechts,

- Einweisung und Behandlung im Rahmen des Unterbringungsrechts,

- Beurteilung sozialrechtlicher Fragen,

- Beurteilung verwaltungsrechtlicher Fragen,

- Beurteilung verkehrsrechtlicher Fragen.

Darüber hinaus war Wissen aus den Nachbargebieten der forensischen Psychiatrie, namentlich aus der Kriminologie, der Rechtspsychologie, dem Prozessrecht, der Rechtsmedizin und der Sexualmedizin nachzuweisen. Besondere Bedeutung wurde dem Wissen und der praktischen Erfahrung bezüglich der Beurteilung und Behandlung spezifischer Störungsbilder in der forensischen Psychiatrie, z. B. aggressives Verhalten, sexuell abweichendes Verhalten, Suizidalität, Intoxikationssyndrome, zugemessen. Spezielles Wissen war auch hinsichtlich der rechtlichen Grundlagen der Beurteilung von Jugendlichen und Heranwachsenden im Straf-, Zivil- und Sorgerecht, der Glaubhaftigkeit von Zeugenaussagen und der Zeugentüchtigkeit, der ethischen und rechtlichen Fragen bei der Diagnostik und Therapie sowie bei der Forschung mit psychisch Kranken und Gestörten nachzuweisen. Die Prinzipien der Qualitätssicherung in der forensischen Psychiatrie mussten theoretisch und praktisch beherrscht werden [14]. Mindestens ein Jahr der Weiterbildungszeit war in einer Maßregelvollzugseinrichtung und ein halbes Jahr in einer auf Begutachtung spezialisierten Einrichtung abzuleisten. Die praktische gutachterliche Erfahrung war durch 70 psychiatrische Gutachten, die unter Supervision selbstständig erstellt und, soweit erforderlich, vor Gericht vertreten wurden, zu belegen.

Im Jahr 2000 wurde der bis dahin wiederholt diskutierte und fortentwickelte Vorschlag zur Reform der Musterweiterbildungsordnung mit dem Schwerpunkt „Forensische Psychiatrie" veröffentlicht [14]. Die gemeinsam mit dem Berufsverband Deutscher Nervenärzte erarbeiteten Vorschläge und die Übergangsbestimmungen wurden im Jahr 2000 unter der Präsidentschaft von H. Süß, Aachen, vom Vorstand der DGPPN gebilligt und im Herbst 2000 etabliert. Es wurde eine auf 3 Jahre befristete Übergangsregelung vom 31.10.2000 bis zum 31.10.2003 für Gebietsärzte geschaffen, die bereits zum Zeitpunkt der Einführung des Curriculums eine ausreichende Qualifikation erworben hatten. Die Zertifizierung im Rahmen der Übergangsregelung setzte eine qualifizierte Fortbildung in forensischer Psychiatrie und Psychotherapie von mindestens 120-h-Dauer, mindestens 3 Jahre fachpsychiatrische oder eine mindestens 6 Jahre lange wissenschaftliche forensisch psychiatrische Tätigkeit voraus. Es waren mindestens 70 selbst erstellte forensisch psychiatrische Falldarstellungen, davon mindestens 50 Gutachten in konkret genannten Bereichen erforderlich sowie mindestens 9 Wochen praktische Fortbildung in einer Maßregelvollzugseinrichtung oder einer stationär psychiatrischen Einrichtung im Justizvollzug; zwei dokumentierte Behandlungsverläufe von psychisch kranken Rechtsbrechern unter kriminalpräventiven Aspekte konnten als praktische Fortbildung anerkannt werden. Die Zertifizierung erfolgte durch einen vom Vorstand der DGPPN eingesetzten Ausschuss. Eine vom Zertifizierungsausschuss benannte Prüfungskommission beurteilte 5 ausgewählte Gutachten [14]. Für den Antrag während der Übergangsregelung waren die Gutachtenliste, die Auflistung „Theoretische Weiterbildung“, Behandlungsberichte, Basisdokumente, ein Curriculum Vitae, die Darstellung wissenschaftlicher Aktivität sowie die Überweisung der Gebühren erforderlich [6]. Nach Ablauf der Übergangsregelung wurden die Anforderungen zum Erwerb der DGPPN-Zertifizierung Forensische Psychiatrie und Psychotherapie aktualisiert. Im Januar 2012 werden gefordert:

- Die Mitgliedschaft in der DGPPN.

- Die Facharztanerkennung für Psychiatrie oder Psychiatrie und Psychotherapie.

- Eine mindestens 3-jährige Fortbildung in forensischer Psychiatrie, davon mindestens 2 Jahre nach der Anerken- 
nung zum Facharzt für Psychiatrie und Psychotherapie oder mindestens 6-jährige forensisch psychiatrische Tätigkeit.

- Der Nachweis von mindestens 240 h theoretischer Ausbildung speziell in forensischer Psychiatrie. Dabei wird eine differenzierte Aufteilung der Inhalte empfohlen.

- Der Nachweis von mindestens 70 eigenen, supervidierten psychiatrischen Gutachten, davon etwa 50 Gutachten zu Fragen des Strafrechts (Schuldfähigkeit und Prognose), 20 zivilrechtliche, sozialrechtliche und Gutachten auf weiteren Rechtsgebieten (Betreuung, Fahreignung). Die Gutachten müssen von einem zertifizierten Psychiater supervidiert werden.

- Ein Jahr klinische Fortbildung in einer akkreditierten Klinik des Maßregelvollzugs oder einer klinisch stationären Einrichtung des Justizvollzugs.

- Das Zertifikat wird nach Überprüfung der Inhalte der theoretischen Weiterbildung und Supervision ausgewählter Gutachten durch den Zertifizierungsausschuss (seit 2011: N. Nedopil, M. Rösler, J.L. Müller) durch die DGPPN erteilt.

Das Zertifikat Forensische Psychiatrie der DGPPN wurde in der Übergangsregelung von zahlreichen Interessenten angenommen. Die Zahl der Inhaber des Zertifikats ist inzwischen auf über 240 Personen angestiegen. Nach Ablauf der Übergangsregelung sank die Nachfrage deutlich und liegt gegenwärtig bei weniger als 10 Anträgen/Jahr. Die aktuelle Liste der durch die DGPPN zertifizierten Gutachter ist über die Website der DGPPN http:/www.dgppn.de/fileadmin/ user_upload/_medien/dokumente/referate/forensische-psychiatrie/ZertifizierteGutachterInnen_DGPPN_04_06_2012. pdf) abrufbar. Die Liste wird laufend aktualisiert; Adressenänderungen sind mitzuteilen.

\section{Weiterbildung der Landesärztekammern}

Im Jahr 2004 wurden die Weiterbildungsordnungen der Landesärztekammern verabschiedet. Sie orientieren sich erkennbar am DGPPN-Zertifikat und sehen ebenfalls eine 36 Monate dauernde praktische Ausbildung vor. Die Ausbildungsordnungen der Landesärztekammern sind bei einer gewissen Heterogenität der Auslegungen weitgehend deckungsgleich. Im Gegensatz zum DGPPN-Zertifikat fordern die Landesärztekammern allerdings weder eine konkrete Zahl von Gutachten, noch werden Gutachten durch erfahrene Supervisoren geprüft. Damit wird aber ein besonders relevanter Anwendungsbereich forensisch psychiatrischer Tätigkeit nicht überprüft.
Gegenwärtige Situation: bessere Qualität und weiteres Entwicklungspotenzial

Mit der Einführung des Zertifikats und der Schwerpunktbezeichnung Forensische Psychiatrie wurden wichtige Maßnahmen zur Qualitätsverbesserung umgesetzt. Das Zertifikat/die Schwerpunktbezeichnung zu erwerben, ist eine wichtige (Begleit-)Motivation für Ärzte geworden, eine Tätigkeit im Maßregelvollzug aufzunehmen. Die Mehrzahl der Sachverständigen hat das Zertifikat/die Schwerpunktbezeichnung bereits erworben oder strebt dies an. Mit zunehmendem Bedarf an Gutachten hat sich auch ein ökonomisch relevantes Geschäftsfeld eröffnet, das die Einkünfte einer wachsenden Zahl von Praxen sichert. Auch dies unterstreicht die Notwendigkeit der regelmäßigen Weiterbildung und Qualitätskontrolle ergänzend und aufbauend auf eine fundierte Ausbildung.

Das Zertifikat Forensische Psychiatrie der DGPPN verstand sich als eine Übergangsregelung der Fachgesellschaft, bis auf Bundes- und Länderebene eine Schwerpunktbezeichnung Forensische Psychiatrie geschaffen wurde. Diese wurde seit 2004 bundesweit eingerichtet. Seitdem steht die Schwerpunktbezeichnung der Landesärztekammern neben dem Zertifikat der DGPPN. Die DGPPN hat mit dem Zertifikat eine Vorreiterrolle bei der Qualitätsverbesserung übernommen und inhaltliche sowie formale Mindestanforderungen eingefordert, die auch von den Ärztekammern zumindest z. T. umgesetzt wurden. Mit der Existenz zweier in der Außenbeurteilung nahezu gleichwertiger Fortbildungen stellt sich angesichts der inzwischen etablierten Schwerpunktbezeichnung auch die Frage, ob das DGPPN Zertifikat Forensische Psychiatrie jetzt, wie ursprünglich intendiert, entbehrlich geworden ist oder inwiefern und mit welcher Zielsetzung das Zertifikat fortentwickelt werden kann, um weitere wichtige Schritte der Qualitätssicherung anzustoßen. Weder ist nämlich die öffentliche Auseinandersetzung um den Stellenwert und die Qualität von Gutachten abgeklungen, noch sind Kritik und Schuldzuweisungen nach Fehlbeurteilungen oder Zwischenfällen verstummt. Unverändert gibt es in Einzelfällen eklatante Qualitätsmängel, die das Ansehen der Expertisen beschädigen (s. dazu auch Süddeutsche Zeitung vom 13.07.2012: diesueddeutsche.de/ bayern/mordfall-vanessa-ein-gutachter-blamiert-sich-vorgericht-1.1411246); angesichts gravierender Qualitätsmängel wurde seitens des Gerichts inzwischen wiederholt die Vergütung zumindest teilweise aberkannt [Begründungen nachzulesen: Juris vom 01.12.2005; Az. 7 StVK 404/10; 7 STVK 296/11 (Juris in press)]; dies betraf auch Träger des DGPPN-Zertifikats. Bemängelt wurde in diesem Zusammenhang, dass auch das DGPPN-Zertifikat ein methodisch und inhaltlich einwandfreies Gutachten nicht gewährleisten kann. 
Abgesehen von diesen Einzelfällen ist die Mehrheit der psychiatrischen Sachverständigen inzwischen auf dem formalen und auch rechtlichen Feld sehr gut geschult und kompetent im Erstatten des Gutachtens. Die fachpsychiatrische Kompetenz in (Differenzial-)Diagnostik, Therapie und Verlaufsbeurteilung ist dagegen in den Hintergrund getreten. Eine lediglich formal korrekte Expertise wird aber dem Gegenstand der Begutachtung nicht gerecht. Inzwischen häufen sich Klagen über lediglich formal korrekte Gutachten, bei denen sich die fachlichen Mängel hinter einer guten Struktur verbergen. Diese Entwicklungen weisen auf die fortbestehende Notwendigkeit der Qualitätsverbesserung hin und werfen die Frage nach einer notwendigen fachinternen Qualitätssicherung auf. Im Interesse der zu begutachtenden Probanden, die einen Anspruch auf eine wissenschaftlich begründete und nachvollziehbare Begutachtung haben, und im Interesse der Auftraggeber ist die DGPPN gefordert, bei diesem notwendigen Prozess neue Impulse zu setzen, die bestehenden Standards fortzuentwickeln und deren verbindliche Einhaltung zu sichern. Nicht zuletzt durch die Forderung, eine Auswahl aus 70 supervidierten Gutachten einem Prüfungsgremium vorzulegen, verfolgt das DGPPN-Zertifikat einen höherwertigeren Anspruch als die Schwerpunktbezeichnung.

Dementsprechend kann das Zertifikat durch die Einführung von Maßnahmen der Qualitätsverbesserung und der Qualitätssicherung fachlich fortentwickelt werden. Damit wird neben der forensischen Basisqualifikation, die in der Schwerpunktbezeichnung der Landesärztekammern erworben wird, eine weitere Qualifikationsstufe geschaffen, die die intensive und fortgesetzte Teilnahme am wissenschaftlichen und fachlichen forensisch psychiatrischen Dialog beinhaltet.

Mit der Fortentwicklung des Zertifikats werden Maßnahmen der Qualitätssicherung und der inhaltlichen Fortentwicklung verankert. Indem regelmäßige Teilnahmen am wissenschaftlichen Fachdialog kontinuierlich nachzuweisen sind und regelmäßige Qualitätskontrollen eingeführt werden, wird die DGPPN-Zertifizierung dynamisiert und an die qualitativ und quantitativ gewachsene Bedeutung forensisch psychiatrischer Expertisen angepasst. Mit der Umsetzung eines kontinuierlichen zertifizierten Fortbildungsprozesses, mit der Formulierung von Qualitätsstandards und der Kontrolle ihrer Einhaltung sowie der Entwicklung von Maßnahmen auf bekannt gewordene Mängel schafft die Fachgesellschaft eine hochwertige, dynamische und aussagekräftige Qualifikation. Durch die Definition konkreter Qualitätsstandards werden die Vorgehensweisen, Beurteilungsgrundlagen und Argumentationsweisen des forensischen Psychiaters für die Justiz als maßgeblichem Auftraggeber der Gutachten transparent dargelegt. Diese Qualitätsstandards orientieren sich an den Mindestanforderungen für die Schuldfähigkeits- und Prog- nosebegutachtung, veröffentlicht von der Interdisziplinären Arbeitsgruppe am Bundesgerichtshof [1,2]. Das DGPPNZertifikat bürgt dann für die kontinuierliche Fortbildung und das Einhalten definierter Qualitätsstandards.

Zusätzlich zu notwendigen Maßnahmen der Qualitätssicherung ist aus fachpsychiatrischer Sicht aber auch zu prüfen, inwiefern bereits niedrigschwellige Anpassungen den Erwerb des Zertifikats erleichtern und damit die Standards forensisch psychiatrischer Tätigkeit insgesamt steigern können. Für allgemeinpsychiatrisch tätige Ärzte hat sich die Ableistung der praktischen Tätigkeit im Maßregelvollzug als problematisch erwiesen, da die intendierte wissenschaftliche bzw. klinische Laufbahn durch den Erwerb der Qualifikation unterbrochen werden muss. Wenngleich die zeitlich befristete praktische Tätigkeit im Maßregelvollzug unverzichtbare Grundlage einer jeden forensisch psychiatrischen Tätigkeit bleibt, können die Ableistung dieses Fortbildungsabschnitts durch den Ausbau von geeigneten Kooperationen und die Einrichtung von Rotationsstellen zwischen allgemeinpsychiatrischen und forensischen Kliniken erleichtert werden. Die enge Vernetzung von allgemeinpsychiatrischer und forensisch psychiatrischer Tätigkeit schafft eine „Win-win“-Situation und erleichtert es den Interessenten, die praktische forensisch psychiatrische Weiterbildung mit ihrem Berufsziel zu vereinbaren. Zugleich profitieren beide Fächer und die beteiligten Kliniken von einem regelmäßigen Austausch.

\section{Maßnahmen der Qualitätsverbesserung}

1. Einholen eines Feedbacks zur Qualitätskontrolle Der Sachverständige erhält nach der Vorlage bzw. nach der Erstattung des Gutachtens keine weiteren Informationen über die Akzeptanz seiner Beurteilung vor Gericht oder das Zutreffen seiner Diagnose, den Erfolg seines Behandlungsvorschlags oder das Eintreffen seiner Prognose. Ein solches Feedback bietet aber eine wesentliche Qualitätssicherungsund Qualitätsverbesserungsoption für die forensisch psychiatrische Tätigkeit. Hierzu können die abgefassten Urteile, Beschlüsse der Strafvollstreckungskammern und Informationen über den weiteren Vollstreckungsverlauf angefordert werden, um zu erfahren,

- ob und inwiefern das Gericht der gutachterlichen Empfehlung folgen konnte,

- ob sich im Fall einer Prognosebegutachtung die Prognose bestätigt hat,

- ob sich die diagnostische Einschätzung für die Unterbringung im Maßregelvollzug als zutreffend erwiesen hat,

- ob die prognostizierte „hinreichend konkrete Erfolgsaussicht" zu einer erfolgreichen Behandlung in der Entziehungsanstalt geführt hat. 
Dagegen sind regelhafte Rückmeldungen über die langfristige Legalbewährung an den Gutachter aus rechtlichen Gründen problematisch. Es wäre aber wünschenswert und für den Gutachter hilfreich, wenn er im Fall erneuter einschlägiger oder anderer gravierender Straftaten von Personen, denen er eine günstige Legalprognose attestiert hat, eine konkrete Rückmeldung zum Vorfall bekäme. Gleiches gilt natürlich, wenn sich schwierige Prognosen eines erfolgreichen Verlaufes bewahrheitet haben.

2. Intervision

Zwischenfälle nach Beurteilungen werden überwiegend als Folge von fehlerhaften Gutachten und als individuelles Versagen gewertet. Eine offene Auseinandersetzung mit der Genese entsprechender Zwischenfälle findet bislang nicht statt. Die offensive, professionelle Diskussion von Zwischenfällen und problematischen Verläufen trägt dazu bei, Fehlerquellen zu erkennen und zu vermeiden. Spezifische Intervisionsseminare und -kolloquien für forensisch psychiatrisch tätige Gutachter und Therapeuten greifen Fälle von falsch-negativen oder falsch-positiven Beurteilungen auf und diskutieren deren Genese in einem geschlossenen kollegialen Rahmen. Nachdem es auch in der forensischen Psychiatrie notwendig ist, auf eine konstruktive, auf Erkenntniszugewinn ausgerichtete Fehlerdiskussionskultur hinzuwirken, ist die Teilnahme an diesen kollegial geführten Intervisionen zu Fehlerquellen bei der Behandlung und Begutachtung besonders zu fördern.

\section{Maßnahmen der Qualitätssicherung}

Nachdem mit der Einführung des Zertifikats und der Schwerpunktbezeichnung Forensische Psychiatrie grundsätzliche Standards geschaffen und etabliert wurden, sind neben der kontinuierlichen inhaltlichen Verbesserung und der zeitgemäßen Anpassung auch Maßnahmen der Qualitätssicherung zu verankern. Diese greifen insbesondere folgendes Problemfeld auf:

Nach Erhalt des Zertifikats werden weitere Fortbildungen nicht mehr in angemessenem Umfang wahrgenommen, sodass der Wissensstand des Gutachters mit dem Zeitpunkt des Erwerbs des Zertifikats stagniert. Zugleich entfernt sich die Qualität der Gutachten eines einmal zertifizierten Sachverständigen zunehmend von den einst geprüften inhaltlichen und formalen Standards. Einzelne Gutachten auch von zertifizierten Sachverständigen werden aufgrund gravierender Qualitätsmängel für nichtgerichtsverwertbar befunden.

Wenn Gutachten auch von zertifizierten Sachverständigen als nichtgerichtsverwertbar bezeichnet und die mit dem Zertifikat verbürgten Qualitätsstandards grob missachtet werden, werden die durchlaufene Weiterbildung und auch die Qualität des Zertifikats entwertet. Dies beschädigt das Zertifikat und gefährdet das Ansehen der forensischen Psychiatrie insgesamt. Gegen eine solche Beschädigung des Zertifikats muss die Fachgesellschaft Maßnahmen zur Qualitätssicherung ergreifen. Die Fachgesellschaft als die für den Standard und die Qualität des Zertifikats bürgende Institution muss in der Lage sein, flexibel auf angeprangerte Missstände zu reagieren und einen kontinuierlichen Prozess der Qualitätskontrolle und Qualitätssicherung zu etablieren. Die hierbei zu diskutierenden Maßnahmen umfassen:

1. die Möglichkeit, auf die Vorlage eklatant mangelhafter Gutachten zu reagieren,

2. eine Ansprechstelle für Gerichte und andere Auftraggeber einzurichten, die bei Zweifel die Qualität vorgelegter Gutachten methodisch beurteilt,

3. die Verstetigung der Weiterbildung im Sinne eines CME-Prozesses.

\section{Schaffung von Reaktionsmöglichkeiten auf gravierende} Qualitätsmängel

Die Fachgesellschaft muss auf die ihr zur Kenntnis gebrachten Qualitätsmängel angemessen und flexibel reagieren können, auch um Ansehen und Renommee des Zertifikats zu schützen. Bei begründeten Zweifeln kann beispielsweise auf Ersuchen des Gerichts nach Vorlage entsprechender Gutachten die Einhaltung der veröffentlichten Qualitätsstandards überprüft werden. Für den Fall gravierender Mängel ist ein Katalog gestufter und geeigneter Maßnahmen vorzubereiten, mit dem angemessen reagiert werden kann. So können zunächst Nachschulungen in Form einer Teilnahme an Fortbildungsveranstaltungen durch die Fachgesellschaft auferlegt werden. $\mathrm{Zu}$ solchen Nachschulungen können insbesondere fachspezifische Wissensdefizite oder gravierende methodische Mängel veranlassen. Die Nachschulungen sollen auf die spezifischen und relevanten Inhalte fokussieren. Nach der fachlichen Überprüfung der im Bedarfsfall Anlass bezogen eingeforderten oder zugesandten Gutachten können systematische Fehler verifiziert, entsprechende Auflagen gemacht und die Behebung der Mängel eingefordert werden. Als Ultima Ratio bei Vorliegen sehr mangelhafter Gutachten muss die Fachgesellschaft dem einmal zertifizierten Sachverständigen das Zertifikat im Extremfall sogar wieder entziehen können. Diese Maßnahmen müssen jedoch stets angemessen, zielführend und dem Zweck entsprechend eingesetzt werden. Maßnahmen der Qualitätskontrolle sind kein Instrument zur Disziplinierung des Sachverständigen; sie sind im Interesse des Sachverständigen grundsätzlich auch geeignet, ungerechtfertigte Zweifel an der Wissenschaftlichkeit des Gutachtens zu entkräften. Der Bedeutung eines solchen fachlichen Kontrollorgans entsprechend muss der DGPPN-Vorstand ein erfahrenes und fachlich ausgewiesenes Expertengremium berufen. Dieses könnte in einem hierzu modifizierten Zertifizierungsausschuss gefunden werden. 
2. Die fachlichen Standards und die inhaltliche Qualität der Gutachten sind durch das Gericht nur bedingt beurteilbar. Wenn seitens des Gerichts oder der Parteien begründete Zweifel an der Wissenschaftlichkeit eines vorgelegten Gutachtens bestehen, kann die für die Standards bürgende Fachgesellschaft auf konkretes Ersuchen die Qualität der erstellten Gutachten überprüfen und methodenkritische Expertisen zur Verfügung stellen. Damit kann die DGPPN verifizieren, ob die methodischen und inhaltlichen Standards, die mit dem Zertifikat bescheinigt werden, eingehalten wurden. Diese Funktion kann den Mitgliedern des oben genannten berufenen Expertengremiums oder eines hierzu modifizierten Zertifizierungsausschusses übertragen werden.

\section{Verstetigung der Weiterbildung im Sinne eines CME-Prozesses}

Zur Fortentwicklung des Zertifikats wird ein kontinuierlicher Fortbildungsprozess entsprechend eines CME-Systems geschaffen. Dieser kann dementsprechend vorsehen, dass das Zertifikat nur noch befristet (z. B. für die Dauer von 5 Jahren) und mit der Auflage, sich regelmäßig weiterzubilden, verliehen wird. Mit der Teilnahme an zertifizierten Veranstaltungen werden Fortbildungspunkte erworben. Unter Nachweis von beispielsweise 200 Punkten, über die in einem Zeitraum von 5 Jahren besuchten forensischen Fortbildungsveranstaltungen, und unter Vorlage einzelner ausgewählter Gutachten wird das Zertifikat um einen weiteren Zeitabschnitt von wiederum 5 Jahren verlängert. Dieser CME-analoge Prozess schließt an die Fortbildungsnachweise der Ärztekammern an. Die abzuleistenden Ausbildungsinhalte sollen dabei nicht nur auf die formal rechtlichen Aspekte fokussieren, sondern auch die fachpsychiatrischen Inhalte in Form von klinischen Seminaren, Supervisionen und Intervisionen einbeziehen.

Mit diesen Maßnahmen wird ein dynamischer Prozess der Qualitätsverbesserung und Qualitätssicherung angestoßen, der im Zusammenwirken mit gleichgerichteten Aktivitäten der Justiz die geforderten Standards und die Praxis forensisch psychiatrischer Expertisen steigern wird. Dies soll durch das fortentwickelte Zertifikat angestoßen werden.

\section{Fazit}

Die DGPPN, die als Organisation das Zertifikat Forensische Psychiatrie verliehen hat, bürgt für die hohe Qualität der Gutachten, die von zertifizierten Sachverständigen erstellt wurden, und nutzt ihre Möglichkeiten der Qualitätssicherung angesichts der gewachsenen Bedeutung forensisch psychiatrischer Expertisen. Die Fortentwicklung des Zertifikats mit Implementierung eines Organs zur Qualitätskontrolle, mit Reaktionsmöglichkeiten auf Qualitätsmängel und Verankerung eines CME-Systems mit befristeter Verleihung und kontinuierlicher Fortbildung setzt die Akzeptanz durch die Sachverständigen ebenso wie die der Auftraggeber voraus. Die Maßnahmen der Qualitätsverbesserung müssen die Auftraggeber (z. B. Gerichte, Staatsanwaltschaften, Justiz) einbeziehen und die für die Auftraggeber relevanten Inhalte adäquat berücksichtigen. Die interdisziplinäre Ausgestaltung schult für die Auftraggeber ausgewiesene Experten, dem aktuellen Stand der Wissenschaft entsprechend; andererseits kennen auch die Auftraggeber die interdisziplinär verankerten Zertifikate und können die angemessene Qualität der Beurteilungen einfordern. Gemeinsam vertretene Standards und kooperativ entwickelte Ausbildungsinhalte verbürgen den interdisziplinären Anspruch forensisch psychiatrischer Expertise. Die Einbeziehung der Auftraggeber in die Entwicklung und Einhaltung gemeinsamer Standards kann am effektivsten dazu beitragen, die Qualität weiter zu steigern und zu sichern.

Interessenkonflikt Es besteht kein Interessenkonflikt.

Open Access Dieser Artikel unterliegt den Bedingungen der Creative Commons Attribution License. Dadurch sind die Nutzung, Verteilung und Reproduktion erlaubt, sofern der/die Originalautor/en und die Quelle angegeben sind.

\section{Literatur}

1. Boetticher A, Nedopil N, Bosinski HAG, SaßH (2005) Mindestanforderungen für Prognosegutachten. Nervenarzt 176:1154-1159

2. Boetticher A, Kröber H-L, Müller-Isberner R, Böhm KM, MüllerMetz R, Wolf T (2006) Mindestanforderungen für Prognosegutachten. NStZ 10(26):537-543

3. Foerster K (1983) Die Forensische Psychiatrie an den Universitäten in der Bundesrepublik Deutschland. Forensia 4:73-9

4. Foerster K (1989) Zur Situation der Weiterbildung in Forensischer Psychiatrie in der Bundesrepublik Deutschland. Nervenarzt 60:243-245

5. Heinz G (1982) Fehlerquellen forensisch-psychiatrischer Gutachten: eine Untersuchung anhand von Wiederaufnahmeverfahren. Kriminalistik, Heidelberg

6. Kröber HL, Müller-Isberner R, Nedopil N, Sass H (2001) DGPPN - Zertifizierung „Forensische Psychiatrie“. Nervenarzt 72:973-974

7. Mende W, Bürke H (1986) Fehlerquellen bei der nervenärztlichen Begutachtung. Forensia 7:143-153

8. Mende W, Schüler-Springorum H (1989) Aktuelle Fragen der forensischen Psychiatrie. In: Kisker KP, Lauter H, Meyer J-E, Müller C, Strömgren E (Hrsg) Psychiatrie der Gegenwart. Springer, Berlin, S 303-337

9. Nedopil N, Müller-Isberner R (1995) Psychiatrischer Maßregelvollzug gemäß $\S 63 \mathrm{StGB}$, Rechtsgrundlage - derzeitige Situation - Behandlungskonzepte - Perspektiven. Nervenarzt 66:793-801

10. Nedopil N, Müller-Isberner JR (1995) Struktur- und Organisationsfragen im psychiatrischen Maßregelvollzug. Monatsschr Kriminol Strafrechtsreform 78:236-244

11. Nedopil N, Sass H (1997) Schwerpunkt forensische Psychiatrie? Nervenarzt 68:529-530

12. Pfäfflin F (1978) Vorurteilsstruktur und Ideologie psychiatrischer Gutachten über Sexualstraftäter. Enke, Stuttgart

13. Rasch W (1967) Schuldfähigkeit. In: Ponsold A (Hrsg) Lehrbuch der gerichtlichen Medizin. Thieme, Stuttgart

14. Sass H (2000) Zur Reform der Musterweiterbildungsordnung. Nervenarzt 71:763-766 